\title{
Loss of Cables, a Novel Gene on Chromosome 18q, in Ovarian Cancer
}

Qun Dong, M.D., Ph.D., Sandra Kirley, B.S., Bo Rueda, Ph.D., Cher Zhao, Lawrence Zukerberg, M.D., Esther Oliva, M.D.

Departments of Pathology (QD, SK, CZ, LZ, EO) and Obstetrics and Gynecology (BR), Massachusetts General Hospital, Harvard Medical School, Boston, Massachusetts

Cables, a cyclin-dependent kinase (cdk) interacting protein, has recently been identified and mapped to human chromosome 18q11. Cables appears to be primarily involved in cell cycle regulation and cell proliferation. Overexpression of Cables in Hela and other cell lines inhibits cell proliferation and tumor formation. We hypothesize that loss of Cables expression is associated with ovarian cancer. To test our hypothesis, we examined Cables expression in the four most common subtypes of ovarian carcinomas: serous, endometrioid, mucinous, and clear cell. In addition, mucinous and serous borderline tumors were also included. Loss of Cables expression was observed at high frequency in ovarian serous (11 of 14 cases, $79 \%$ ) and endometrioid (5 of 10 cases, $50 \%$ ) carcinomas. In contrast, strong Cables staining was detected in all clear cell carcinomas (10 cases) and mucinous tumors (5 carcinomas and 5 borderline tumors). The majority of serous borderline tumors ( 11 of 14 cases, $79 \%$ ) showed positive Cables staining, with the rest showing focal loss of Cables expression. Furthermore, RT-PCR revealed the lack of Cables mRNA in a human ovarian cancer xenograft. No correlation was noted between loss of Cables and histologic grade, tumor stage, and survival. In conclusion, our results indicate that loss of Cables is common in ovarian serous and endometrioid carcinomas and imply that Cables may be involved in the pathogenesis of these two types of ovarian carcinomas.

KEY WORDS: Cables, Ovarian cancer, Ovary, Tumorigenesis.

Mod Pathol 2003;16(9):863-868

Copyright (c) 2003 by The United States and Canadian Academy of Pathology, Inc.

VOL. 16, NO. 9, P. 863, 2003 Printed in the U.S.A.

Date of acceptance: May 23, 2003.

Address reprint requests to: Esther Oliva, M.D., Department of Pathology,

Massachusetts General Hospital, Harvard Medical School, 55 Fruit Street,

Warren 251A, Boston, MA 02114; e-mail: eoliva@partners.org.

DOI: 10.1097/01.MP.0000084434.88269.0A
Molecular analysis of allelic loss or loss of heterozygosity ( $\mathrm{LOH})$ has been widely used to delineate the chromosomal locations of putative tumor suppressor genes involved in tumorigenesis (1). Studies on ovarian cancer have demonstrated $\mathrm{LOH}$ at numerous chromosomal locations including $3 p, 5 q, 6,7 p$, $8,9 q, 11 p, 13 q, 17,18 q, 21 q$, and 22q (2-5). Loss or deletion of chromosome $18 \mathrm{q}$ is one of the common chromosomal abnormalities in ovarian cancer, and has been reported to occur in 25 to $40 \%$ of ovarian carcinomas (2-3). Cables was initially identified and characterized as a link between Cdk5 and c-Abl to promote neurite outgrowth (6). Subsequent studies mapped the Cables gene to chromosome 18q11-12 and demonstrated that Cables may serve as a putative mediator of the cell cycle and a potential tumor suppressor $(7,8)$. Therefore, deletion in chromosome 18q in ovarian cancer could lead to loss of Cables expression.

Cables acts as a link connecting proteins, most notably the cyclin-dependent kinases (cdks) and non-receptor tyrosine kinases, such as c-Abl or Weel. The cdks comprise a family of serine/threonine protein kinases that regulate cell cycle progression. Alteration of cdk functions can lead to cancer development. Some of the cdk-interacting proteins, such as p16 and p21, act as tumor suppressor genes or direct downstream targets of tumor suppressor genes $(9,10)$. Loss of these proteins is frequent in primary human cancers. Cables inhibits cdk2 activity by enhancing Weel-mediated inhibitory tyrosine phosphorylation (8) and therefore may lead to slowed cell cycle progression. Moreover, overexpression of Cables in cell lines inhibits cell proliferation and prevents tumor formation in nude mice (Zukerberg, unpublished data). Furthermore, the Cables knockout mouse develops early carcinoma of the endometrium at 3 months of age (Zukerberg, unpublished observations). These results support the hypothesis that Cables may be involved in regulation of cell proliferation, and loss of Cables may cause uncontrolled cell proliferation and promote tumorigenesis. 


\section{MATERIAL AND METHODS}

\section{Immunohistochemistry}

GST-Cables fusion proteins were expressed in Escherichia coli and purified with GSH coupled beads. A specific antibody was raised against a GSTtagged human Cables in rabbits and purified by affinity column. Formalin-fixed, paraffin-embedded sections of normal and tumor tissue, which had IRB approval, were stained with affinity purified polyclonal anti-Cables antisera, at a 1:200 dilution, using a microwave-enhanced avidin-biotin staining method (11). Negative control sections were immunostained under the same conditions, substituting preabsorbed antisera and preimmune rabbit antisera for primary antibodies. The specificity of the affinity-purified antisera was demonstrated by lack of staining with preabsorbed and preimmune antisera, and strong staining of COS7 cells transfected with Cables with little to no staining of nontransfected cells, which contained few Cables by Western blot (8).

The nuclear expression of Cables was assessed by immunohistochemical staining on formalin-fixed, paraffin-embedded tissue sections of 28 serous tumors ( 14 carcinomas, and 14 borderline tumors), 10 mucinous tumors ( 5 carcinomas and 5 borderline tumors), 10 endometrioid carcinomas, and 10 clear cell carcinomas. Complete loss of Cables was defined as negative nuclear staining of all the tumor cells, with positive nuclear staining of associated normal ovarian tissue including fibroblasts and inflammatory cells. Partial loss of Cables was defined as both negative and unequivocal positive stained tumor cells in the same tissue section, despite even staining of background normal tissue. All cases were evaluated by three pathologists (QD, LZ, EO).

In addition, immunohistochemical stains for p53 and MIB1 were performed in the 14 serous carcinomas using the avidin-biotin peroxidase technique. Paraffin-embedded sections were deparaffinized and heated in a pressure cooker at $120^{\circ} \mathrm{C}$ for 5 minutes for antigen retrieval. The sections were subsequently incubated for 5 minutes in 3\% hydrogen peroxide to quench endogenous peroxidase activity. The sections were then incubated with the primary antibodies (1:15 dilution for anti-p53 and 1:40 for anti-MIB1), followed by secondary antibodies and avidin-biotin staining. The intensity of p53 staining was graded as negative, weakly positive, positive, and strongly positive.

\section{cDNA Expression}

The Cables mRNA of human tumor xenografts was studied using a panel of 7 normalized, firststrand cDNA preparations from human tumor xenografts (Human Tumor MTC Panel, Clontech), according to the manufacturer's directions. Cables PCR primers were 5'-GCAGGAGGACTGTGGCCTTG AGGAG-3', 5'-CTGTGTGCTGGGGCATGTGTG -CTG T-3' and 3'-GGCCCTTGGCTGTCCTCGGGGCCAGT G-5', which amplified the very $\mathrm{COOH}$-terminal 350 bp and $250 \mathrm{bp}$ of the open reading frame. G3PDH PCR primers (included in the manufacturer's kit) were used as a cDNA normalization control.

\section{RESULTS}

\section{Cables Expression in Normal Ovarian Tissue}

Normal ovarian surface epithelium (Fig. 1A), ovarian stromal cells (Fig. 1B), and adherent fallopian tube epithelium (Fig. 1C) showed strong nuclear staining. In addition, inflammatory cells including lymphocytes and plasma cells, when present, were positive for Cables staining as well.

\section{Cables Expression in Ovarian Cancers}

Strong Cables nuclear staining was observed in all mucinous borderline tumors and carcinomas (Fig. 2A) and clear cell carcinomas (Fig. 2B). In contrast, 11 of 14 serous carcinomas (79\%) showed complete (9 cases; Fig. 3A) or partial (2 cases) loss of Cables nuclear staining. The remaining 3 tumors showed positive Cables nuclear staining (Fig. 3B). Among the 14 borderline serous tumors, 11 showed positive Cables staining, and the other 3 showed partial loss of Cables. Five of 10 endometrioid carcinomas (50\%) showed complete (3 cases, Fig. 4A) or partial ( 2 cases) loss of Cables staining, whereas the other 5 showed positive nuclear Cables expression (Fig. 4B). The benign surface epithelium and background stromal cells as well as inflammatory cells adjacent to tumor cells, when present, showed positive nuclear staining.

All 14 serous carcinomas showed strong positive p53 staining, as well as relatively high percentage of MIB1-positive cells (20-80\%). There was no statistically significant difference in the number or intensity of p53 and MIB1 staining between Cablespositive and -negative serous carcinomas.

\section{Clinical and Pathological Findings}

As serous and endometrioid ovarian carcinomas showed variable nuclear expression of Cables, we tried to further evaluate any possible correlation among Cables-positive and -negative tumors and clinicopathologic features associated with these tumors (see Tables 1 and 2). No correlation was noted between loss of nuclear Cables staining and histologic grade, clinical stage, or outcome among the serous or endometrioid carcinomas. 


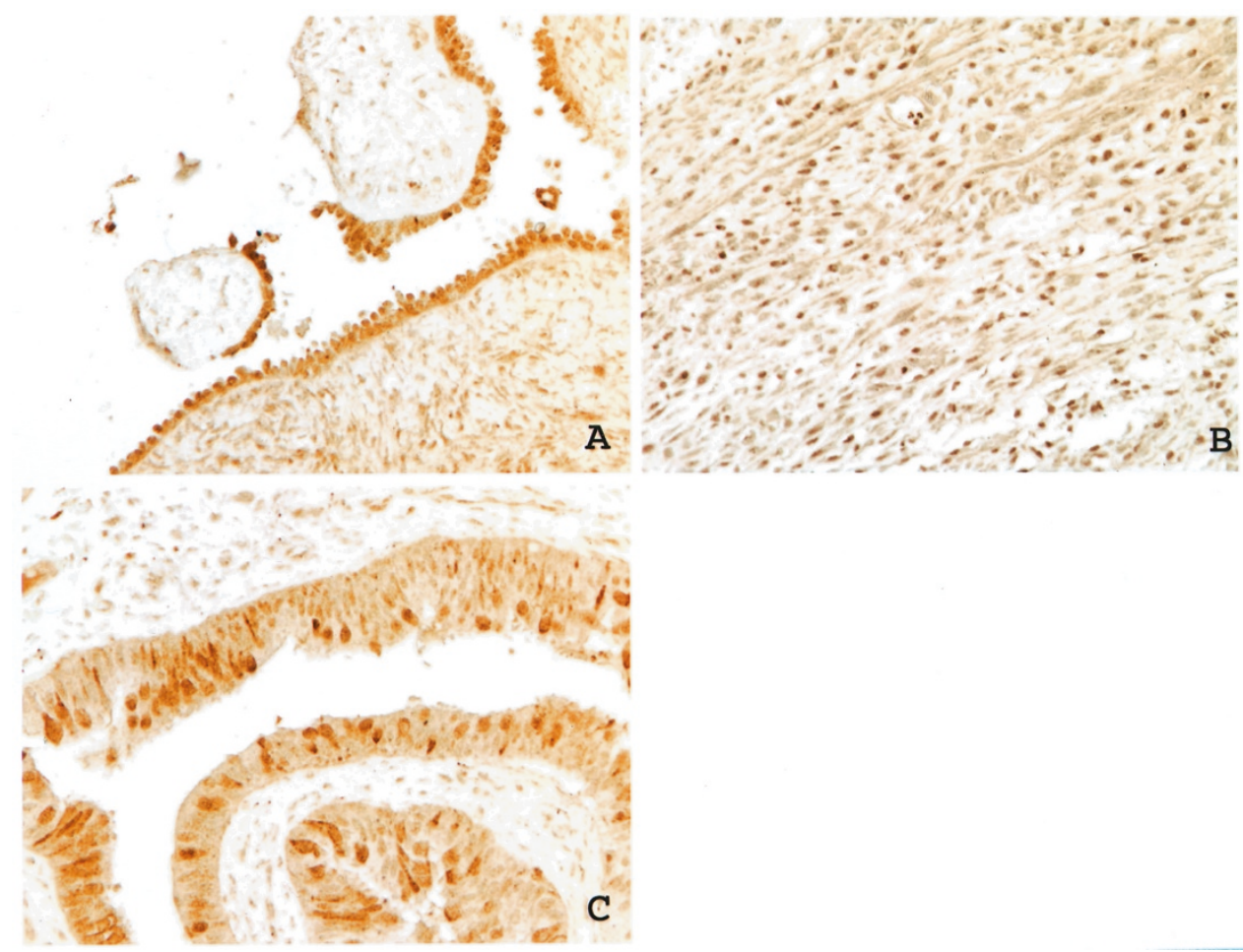

FIGURE 1. Strong nuclear Cables staining in ovarian surface epithelium (A), ovarian stromal cells (B), and fallopian tube epithelium (C).
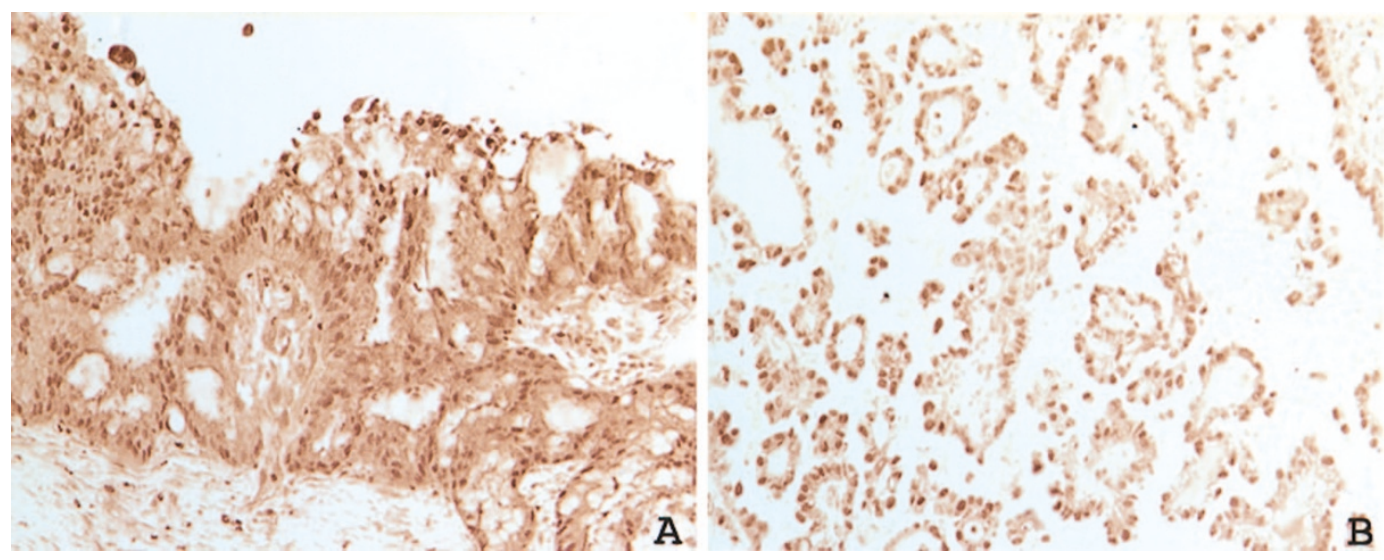

FIGURE 2. Strong nuclear Cables reactivity in ovarian mucinous carcinoma (A) and clear cell carcinoma (B).

\section{Cables cDNA Expression}

First-strand cDNA from seven human tumor xenografts (Clontech), including lung, colon, breast, pancreas, and ovary tumors were screened with Cables-specific primers from the very $\mathrm{COOH}$ terminal portion of human Cables. Four tumors showed approximately equal levels of Cables cDNA, whereas three tumors (colon, lung, and ovary) showed markedly decreased Cables cDNA (Fig. 5). G3PDH cDNA levels were the same in all of the tumors. These results support loss of Cables at the mRNA level in ovarian cancer.

\section{DISCUSSION}

Chromosomal 18q loss is a common event in ovarian cancer. Most of the research has been focused on the distal portion of 18q, where previously characterized putative tumor suppressor genes DCC, SMAD4, and SMAD2 are located. However, a significant number of ovarian cancers show loss of alleles in both the proximal and distal portions (3, 4). Furthermore, there are carcinomas that show loss of alleles in only the proximal region of 18q, which does not include DCC, SMAD4, and SMAD2 

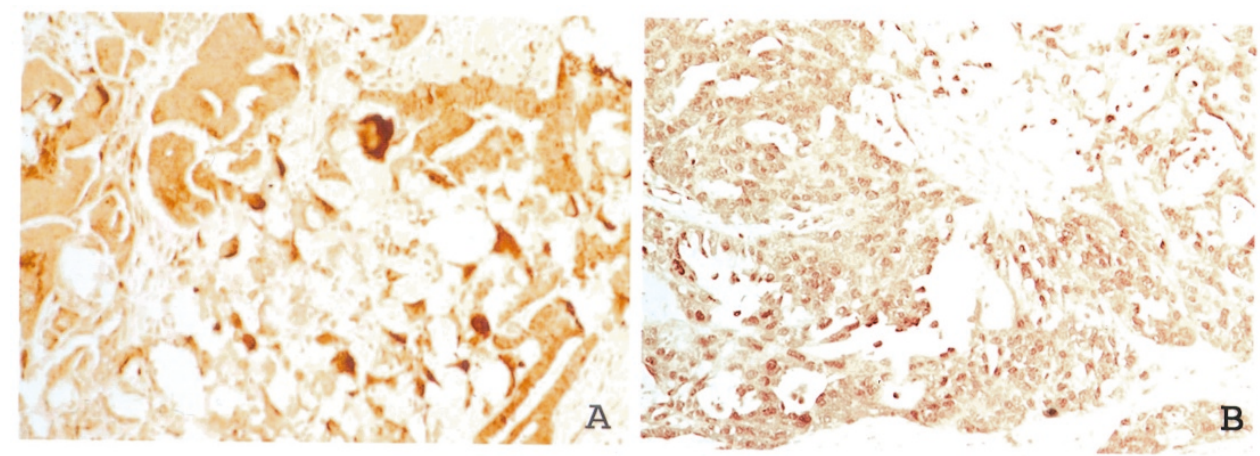

FIGURE 3. Expression of Cables in ovarian serous carcinoma. Negative (A) and positive (B) nuclear Cables staining in different cases.

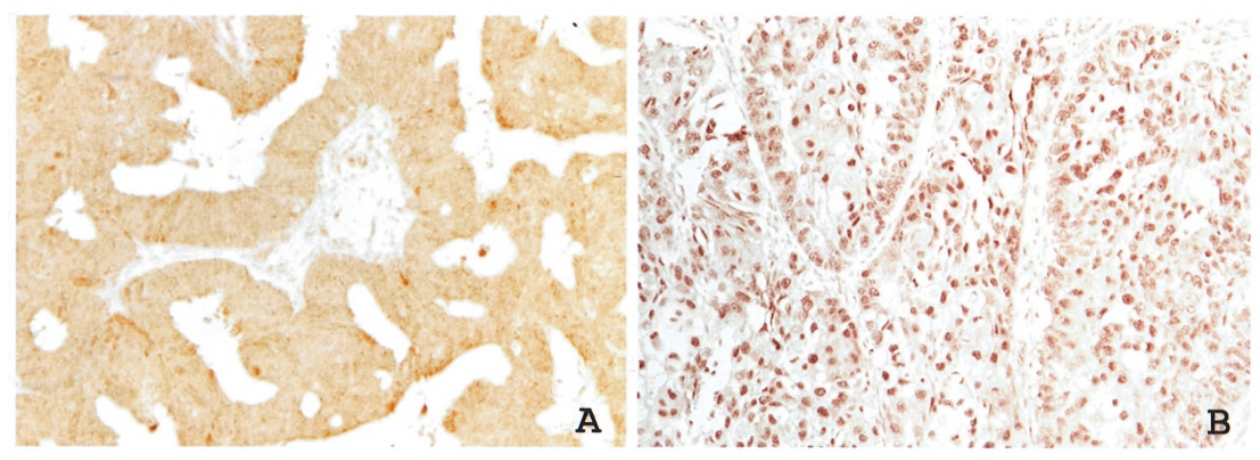

FIGURE 4. Negative (A) and positive (B) nuclear expression of Cables in ovarian endometrioid carcinoma.

TABLE 1. Correlation of Cables Expression with Clinicopathologic Findings of Ovarian Serous Carcinoma

\begin{tabular}{ccccc}
\hline $\begin{array}{c}\text { Age } \\
\text { (years) }\end{array}$ & $\begin{array}{c}\text { Histologic } \\
\text { Grade }\end{array}$ & Stage & Follow-Up & $\begin{array}{c}\text { Cables } \\
\text { Staining }\end{array}$ \\
\hline 62 & $3 / 3$ & T3cN0M0 & DOD & $-/+$ \\
71 & $3 / 3$ & T3cN1M0 & DOD & - \\
42 & $3 / 3$ & T3cN0M0 & AWD & - \\
32 & $3 / 3$ & T3cN1M1 & DOD & - \\
78 & $3 / 3$ & T3cN1M0 & DOD & - \\
74 & $3 / 3$ & T3cN1M0 & DOD & + \\
59 & $3 / 3$ & T3cN0M0 & DOD & - \\
49 & $3 / 3$ & T3cN0M1 & DOD & - \\
36 & $3 / 3$ & T3cN1M0 & DOD & - \\
48 & $3 / 3$ & T3cN0M0 & DOD & + \\
74 & $3 / 3$ & T3bN1M0 & AWD & - \\
51 & $3 / 3$ & T3cN0M1 & DOD & - \\
60 & $3 / 3$ & T3N0M1 & DOD & + \\
40 & $3 / 3$ & T3cN1M1 & DOD & $-/+$ \\
\hline
\end{tabular}

AWD: alive with disease; DOD: died of disease; $-1+$ : partial loss of staining.

(3). Supporting this data is the fact that DCC is now known to be a netrin receptor, and mutations or losses occur in only a very small number of human tumors (12). Similarly, although SMAD4 is important in pancreatic cancer, few mutations or cases with biallelic inactivation have been found outside the pancreas. These studies imply that there might be other potential tumor suppressor genes in the proximal region of $18 \mathrm{q}$ that may be involved in
TABLE 2. Correlation of Cables Expression with Clinicopathologic Findings of Ovarian Endometrioid Adenocarcinoma

\begin{tabular}{ccllc}
\hline $\begin{array}{c}\text { Age } \\
\text { (years) }\end{array}$ & $\begin{array}{c}\text { Histologic } \\
\text { Grade }\end{array}$ & Stage & Follow-Up & $\begin{array}{c}\text { Cables } \\
\text { Staining }\end{array}$ \\
\hline 81 & $2 / 3$ & T1aN0M0 & AW & $-/+$ \\
68 & $2 / 3$ & T1cN0M0 & AW & + \\
60 & $2 / 3$ & T1cN0M0 & AW & - \\
82 & $3 / 3$ & T3bN0M0 & DOD & $-/+$ \\
41 & $3 / 3$ & T1bN1M0 & DOD & + \\
57 & $1 / 3$ & T1bN0M0 & AW & + \\
41 & $2 / 3$ & T1bN0M0 & AW & - \\
80 & $3 / 3$ & T1cN0M0 & AW & - \\
69 & $2 / 3$ & T1cN0M0 & DOD & - \\
54 & $2 / 3$ & T1aN0M0 & AW & - \\
\hline
\end{tabular}

AW: alive well; DOD: died of disease; $-/+$ : partial loss of staining.

ovarian tumorigenesis. As the genetic locus of Cables is $18 \mathrm{q} 11-12$ and its primary function appears to be regulating cell proliferation, Cables could be a candidate tumor suppressor gene in the proximal region of 18q. This idea is supported by our studies on colorectal adenocarcinoma and head and neck squamous cell carcinoma (8), which revealed that loss of Cables correlated with LOH on 18q11-12.

To study the potential role of Cables in ovarian cancer, we examined the expression of Cables in the four most common types of ovarian epithelial carcinomas, as well as in mucinous and serous 

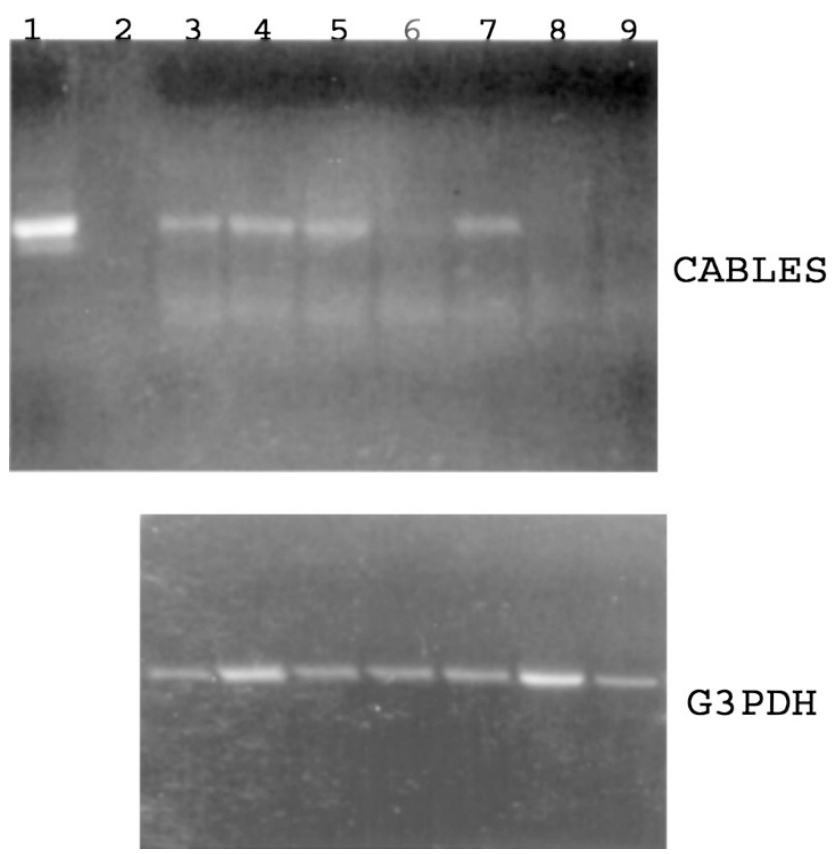

FIGURE 5. Detection of Cables mRNA levels by RT-PCR. Cables mRNA expression is detected in breast cancer (Lane 3), pancreatic carcinoma (Lane 7), one lung cancer (Lane 4), and one colon cancer (Lane 5). Markedly decreased Cables mRNA expression is detected in ovarian cancer (Lane 9), one lung cancer (Lane 8), and one colon cancer (Lane 6). Lane 1, Cables cDNA positive control. Lane 2, blank. Lower panel, RT-PCR with G3PDH as a control for normalization of the mRNA levels.

borderline tumors. Loss of Cables was evident in a high percentage of ovarian serous and endometrioid carcinomas. Furthermore, loss of Cables was also observed at the mRNA level by RT-PCR analysis in a human ovary tumor xenograft. In contrast to serous and endometrioid carcinomas, all clear cell carcinomas, mucinous borderline tumors and carcinomas, and the majority of the serous borderline tumors showed strong Cables nuclear staining. These results indicate that Cables may be involved in the development of ovarian serous and endometrioid carcinomas and suggest that the tumorigenesis of clear cell and mucinous tumors may be mediated through mechanisms that are different from serous and endometrioid carcinomas. Moreover, our findings support the hypothesis that serous borderline tumor is a separate entity and does not appear to be a required previous step for the development of serous carcinoma (13-15).

Loss of Cables expression has also been observed in other cancers, including colorectal adenocarcinoma, head and neck squamous cell carcinoma, lung squamous cell carcinoma, esophageal squamous cell cancer, endometrial endometrioid carcinoma, and bladder transitional cell carcinoma. RTPCR analysis confirmed loss of Cables mRNA in ovarian, colon, and lung cancers, which correlates with results of previous studies (8). In contrast to these carcinomas, loss of Cables is uncommon in pancreatic and breast cancers. Although loss of $18 \mathrm{q}$ occurs in $\geq 90 \%$ of pancreatic adenocarcinoma (16), loss of Cables occurs only in $12.5 \%$ of pancreatic cancer (Zukerberg, unpublished observations). Therefore, loss of Cables expression is not always associated with $\mathrm{LOH}$ on 18q.

Loss of Cables in ovarian cancer could lead to uncontrolled cell proliferation through different mechanisms. Cables interacts with many proteins involved in cell proliferation, including cdk2 and p53. Cables enhances a cdk2-inhibitory phosphorylation by the Weel protein kinase (8), which decreases cdk2 activity. Therefore loss of Cables may disregulate cdk2 function and enhance tumor formation. In addition, it is well characterized that almost all tumors develop resistance to growth inhibitory signals, and genes involved in this resistance almost always relate to the retinoblastoma gene product, $\mathrm{pRB}$. Mutations in $\mathrm{pRB}$, viral infections, and alteration of genes that normally regulate $\mathrm{pRB}$ are common mechanisms of $\mathrm{pRB}$ inactivation (17). Because pRB is regulated by cdks, loss of Cables, which leads to disregulated cdk2 activity, would also cause pRB inactivation.

Finally, p53 mutation is a common event in ovarian serous carcinomas $(18,19)$. Cables interacts with p53 and may enhance p53-induced apoptosis (20). To test whether there was a correlation between p53 mutations and Cables loss, we studied p53 expression in serous carcinomas. All serous carcinomas in our series were positive for p53 regardless of Cables expression. These results imply that loss of Cables might be a late event in tumorigenesis. Further studies will be helpful to determine more specifically the role of Cables in the timeline of ovarian tumor development. No significant difference in MIB1-positive cells was detected between Cables-positive and -negative serous carcinomas in our series. No correlation of Cables expression versus tumor grade, stage, or survival was seen. However, the number of cases in this study, as well as the fact that most carcinomas were high grade, makes it difficult to draw definitive conclusions about the possible correlation.

In summary, our data provide evidence that loss of Cables is associated with ovarian serous and endometrioid carcinomas and suggest that Cables may be involved in the pathogenesis of these two types of carcinomas. Mutational analysis of the $\mathrm{Ca}$ bles gene in ovarian cancer and evaluation of the ovary in Cables knockout mice are currently underway to further characterize the role of Cables in ovarian development and tumorigenesis.

\section{REFERENCES}

1. Ponder B. Cancer gene losses in human tumours. Nature 1988;335:400-3. 
2. Cliby W, Ritland S, Hartmann L, Dodsom M, Halling KC, Keeney G, et al. Human epithelial ovarian cancer allelotype. Cancer Res 1993;53:2393-8.

3. Arnold N, Hagele L, Walz L, Schempp W, Pfisterer J, Bauknecht $\mathrm{T}$, et al. Overrepresentation of $3 \mathrm{q}$ and $8 \mathrm{q}$ material and loss of $18 \mathrm{q}$ material are recurrent findings in advanced human ovarian cancer. Genes Chromosomes Cancer 1996;16:46-54.

4. Pere H, Tapper J, Seppala M, Knuutila S, Butzow R. Genomic alterations in Fallopian tube carcinoma: comparison to serous uterine and ovarian carcinomas reveals similarity suggesting likeness in molecular pathogenesis. Cancer Res 1998; 58:4274-6.

5. Lassus H, Salovaara R, Arltonen LA, Butzow R. Allelic analysis of serous ovarian carcinoma reveals two putative tumor suppressor loci at 18q22-q23 distal to SMAD4, SMAD2, and DCC. Am J Pathol 2001;159:35-42.

6. Zukerberg LR, Patrick GN, Nikolic M, Humbert S, Wu CL, Lanier LM, et al. Cables links cdk5 and c-Abl and facilitates cdk5 tyrosine phosphorylation, kinase upregulation, and neurite outgrowth. Neuron 2000;26:633-46.

7. Matsuoka M, Matsuura Y, Semba K, Nishimoto I. Molecular cloning of a cyclin-like protein associated with cyclin dependent kinase 3 (cdk3) in vivo. Biochem Biophys Res Commun 2000;273:442-7.

8. Wu CL, Kirley SD, Xiao H, Chuang Y, Chung DC, Zukerberg LR. Cables enhances cdk2 tyrosine 15 phosphorylation by Wee1, inhibits cell growth, and is lost in many human colon and squamous cancers. Cancer Res 2001;61:7325-32.

9. Bonetta L. Open questions on p16. Nature 1994;370:180.

10. Xiong Y, Hannon GJ, Zhang H, Casso D, Kobayashi R, Beach D. p21 is a universal inhibitor of cyclin kinases. Nature 1993;366:701-4.

11. Hsu SM, Raine L, Fanger H. Use of avidin-biotin-peroxidase complex $(\mathrm{ABC})$ in immunoperoxidase techniques: a compar- ison between $\mathrm{ABC}$ and unlabeled antibody (PAP) procedures. J Histochem Cytochem 1981;29:577-80.

12. Gotley DC, Reeder JA, Fawcett J, Walsh MD, Bates P, Simmons DL, et al. The deleted in colon cancer (DCC) gene is consistently expressed in colorectal cancers and metastases. Oncogene 1996;13:787-95.

13. Ortiz BH, Ailawadi M, Colitti C, Muto MG, Deavers M, Silva $\mathrm{EG}$, et al. Second primary or recurrent? Comparative patterns of p53 and k-ras mutations suggest that serous borderline ovarian tumors and subsequent serous borderline ovarian tumors and subsequent serous carcinomas are unrelated tumors. Cancer Res 2001;61:7264-7.

14. Singer G, Kurman RJ, Chang HW, Cho SK, Shih IEM. Diverse tumorigenic pathways in ovarian serous carcinoma. Am J Pathol 2002;160:1223-8.

15. Feeley KM, Wells M. Precursor lesions of ovarian epithelial malignancy. Histopathology 2001;38:87-95.

16. Hahn SA, Hoque A, Moskaluk CA, da Costa L, Schutte M, Rozenblum E, et al. Homozygous deletion map at 18q21.1 in pancreatic cancer. Cancer Res 1996;56:490-4.

17. Zukerberg L. The molecular basis of dysplasia. Semin Diagn Pathol 2002;19:48-53.

18. Werness BA, Freedman AN, Piver MS, Romero-Gutierrez M, Petrow E. Prognostic significance of p53 and p21 $1^{\text {wafl/cip } 1}$ immunoreactivity in epithelial cancers of the ovary. Gynecol Oncol 1999;75:413-8.

19. Geisler JP, Geisler HE, Miller GA, Wiemann MC, Zhou Z, Crabtree W. p53 and bcl-2 in epithelial ovarian carcinoma: their value as prognostic indicators at a median follow-up of 60 months. Gynecol Oncol 2000;77:278-82.

20. Tsuji K, Mizumoto K, Yamochi T, Nishimoto I, Matsuoka M. Differential effect of ik3-1/cables on p53- and p73-induced cell death. J Biol Chem 2002;277:2951-7. 